\title{
Fabrication of GaOx Confinement Structure for InGaN Light Emitter Applications
}

\author{
Yi-Yun Chen ${ }^{1}$, Yuan-Chang Jhang ${ }^{1}$, Chia-Jung Wu ${ }^{1}$, Hsiang Chen ${ }^{2}$, Yung-Sen Lin ${ }^{3}$ and \\ Chia-Feng Lin $1, *$ iD \\ 1 Department of Materials Science and Engineering, Innovation and Development Center of Sustainable \\ Agriculture, Research Center for sustainable energy and Nanotechnology, National Chung Hsing University, \\ No. 145, Xingda Road, South Dist., Taichung 402, Taiwan; singyi.sky@gmail.com (Y.-Y.C.); \\ d9883122@gmail.com (Y.-C.J.); thulongwu@gmail.com (C.-J.W.) \\ 2 Department of Applied Materials and Optoelectronic Engineering, National Chi Nan University, No. 1, \\ University Road, Puli Township, Nantou County 545, Taiwan; hchen@ncnu.edu.tw \\ 3 Department of Chemical Engineering, Feng Chia University, No. 100, Wenhwa Road, Seatwen, \\ Taichung 40724, Taiwan; yslin@fcu.edu.tw \\ * Correspondence: cflin@dragon.nchu.edu.tw; Tel.: +886-2284-0500 (ext. 706)
}

Received: 29 September 2018; Accepted: 4 November 2018; Published: 7 November 2018

\begin{abstract}
An indium gallium nitride (InGaN) light-emitting diode (LED) with an embedded porous $\mathrm{GaN}$ reflector and a current confined aperture is presented in this study. Eight pairs of $\mathrm{n}^{+}-\mathrm{GaN}: \mathrm{Si} / \mathrm{GaN}$ in stacked structure are transformed into a conductive, porous GaN/GaN reflector through an electrochemical wet-etching process. Porous GaN layers surrounding the mesa region were transformed into insulating $\mathrm{GaOx}$ layers in a reflector structure through a lateral photoelectrochemical (PEC) oxidation process. The electroluminescence emission intensity was localized at the central mesa region by forming the insulating $\mathrm{GaOx}$ layers in a reflector structure as a current confinement aperture structure. The PEC-LED structure with a porous GaN reflector and a current-confined aperture surrounded by insulating $\mathrm{GaOx}$ layers has the potential for nitride-based resonance cavity light source applications.
\end{abstract}

Keywords: InGaN; porous GaN; insulating GaOx; current confinement aperture structure

\section{Introduction}

Gallium nitride (GaN) materials have been developed for optoelectronic devices such as lightemitting diodes (LEDs), laser diodes (LD) [1], and vertical cavity surface emitting lasers (VCSEL) [2]. Lateral oxidation of high aluminum (Al) content AlGaAs-based epilayers [3,4] produces a currentconfining aperture in the central microcavity structure. The lateral oxidized process was used to transform AlAs into insulating AlOx, acting as a current confinement aperture in the microcavity structure [5]. For nitride materials, Dorsaz et al. [6] reported the use of the electrochemical oxidation process for AlInN to form current apertures. The epitaxial AlGaN/GaN stack [7], AlN/GaN stacks [8,9], and AlInN/GaN stack [10] structures have been reported for bottom epitaxial distributed Bragg reflector (DBR) in GaN-based VCSEL devices. Here, an InGaN-based LED structure with $\mathrm{n}-\mathrm{GaN}: \mathrm{Si} / \mathrm{GaN}$ stack epi-layers is transformed into a conductive porous-GaN/GaN reflector through a wet electrochemical (EC) etching process. Then, the porous GaN layers surrounding the mesa region are transformed into insulating $\mathrm{GaOx}$ layers in the stack structure through a photoelectrochemical (PEC) oxidation process. A current-confined aperture in the bottom porous GaN reflector was produced in the central mesa region. 


\section{Experimental}

The LED epitaxial layer consisted of a $30 \mathrm{~nm}$-thick GaN buffer layer grown at $530^{\circ} \mathrm{C}$, a $2.0 \mu \mathrm{m}$-thick unintentionally doped GaN layer (u-GaN, $\left.1050^{\circ} \mathrm{C}, 5 \times 10^{16} \mathrm{~cm}^{-3}\right)$, a $1.0 \mu \mathrm{m}$-thick n-GaN layer $\left(1050^{\circ} \mathrm{C}\right.$, $\left.2 \times 10^{18} \mathrm{~cm}^{-3}\right)$ for the bottom $n$-type contact layer, eight pairs of $n^{+}-\mathrm{GaN}: \mathrm{Si} / \mathrm{GaN}\left(\mathrm{n}^{+}-\mathrm{GaN}: \mathrm{Si}\right.$ with $2 \times$ $\left.10^{19} \mathrm{~cm}^{-3}\right)$ stack structure, a $0.2 \mu \mathrm{m}$-thick u-GaN layer $\left(1050^{\circ} \mathrm{C}\right)$, a $0.3 \mu \mathrm{m}$-thick $\mathrm{n}$-GaN layer $\left(1050{ }^{\circ} \mathrm{C}\right.$, $2 \times 10^{18} \mathrm{~cm}^{-3}$ ), six pairs of $\operatorname{In}_{0.2} \mathrm{GaN} / \mathrm{GaN}\left(3 \mathrm{~nm} / 12 \mathrm{~nm}\right.$ ) multiple quantum wells (MQWs, $760{ }^{\circ} \mathrm{C}$ ), and a $0.1 \mu \mathrm{m}$-thick p-GaN layer $\left(950^{\circ} \mathrm{C}, 1 \times 10^{18} \mathrm{~cm}^{-3}\right)$. A $150 \mathrm{~nm}$-thick indium tin oxide (ITO) film was deposited on the mesa region and acted as a transparent conductive layer. The mesa regions of the InGaN LED structures were defined using a laser scribing (LS) process and dry etching process with a $1.5 \mu \mathrm{m}$ etching depth to produce the as-grown eight-period $\mathrm{n}^{+}-\mathrm{GaN} / \mathrm{GaN}$ stack structure. The LED chips were $35 \times 35 \mu^{2}$ in size, and the mesa regions were defined by using a triple frequency ultraviolet $\mathrm{Nd}: \mathrm{YVO}_{4}(355 \mathrm{~nm})$ laser for the front side laser scribing process with a $40 \mu \mathrm{m}$ spacing width. The samples were immersed in a $0.5 \mathrm{M}$ nitride acid solution for wet electrochemical (EC) etching with an external direct current (DC) bias voltage of $+8 \mathrm{~V}$. The EC-treated LED structure with a porous GaN reflector was defined as an EC-LED. Then, the samples were oxidized through a PEC oxidation process with a $+20 \mathrm{~V}$ bias voltage and illuminated with a $400 \mathrm{~W} \mathrm{Hg}$ lamp in deionized water for $30 \mathrm{~min}[11,12]$. The porous $\mathrm{GaN} / \mathrm{GaN}$ stack reflector structure was transformed into a $\mathrm{GaOx} / \mathrm{GaN}$ stack reflector. The PEC-treated LED with $\mathrm{GaOx} / \mathrm{GaN}$ stack structure surrounding the mesa region was defined as a PEC-LED. The electroluminescence (EL) spectra were measured through an optical spectrum analyzer (iHR550, Edison, NJ, USA) and the light intensity profiles were measured by a beam profiler (Spiricon, Jerusalem, Israel).

\section{Results and Discussions}

The optical microscopy (OM) images of the standard-LED (ST-LED), electrochemical etch LED (EC-LED), and photoelectrochemical oxidized LED (PEC-LED) structures are shown in Figure 1a-c, respectively. After the EC wet etching process, the $\mathrm{n}^{+}-\mathrm{GaN}: \mathrm{Si} / \mathrm{u}-\mathrm{GaN}$ stack structure was transformed into a porous GaN/GaN reflector as shown in Figure 1b. The $\mathrm{n}^{+}-\mathrm{GaN}: \mathrm{Si}$ layers in the stack structure were etched to form the porous $\mathrm{GaN}$ layers. The laser scribing line patterns provided the wet etching channels on the $\mathrm{n}^{+}-\mathrm{GaN}: \mathrm{Si} / \mathrm{GaN}$ stack structure. The porous $\mathrm{GaN}$ reflector in the blue light range was observed on the mesa region. Then, the porous $\mathrm{GaN} / \mathrm{GaN}$ reflector was transformed into a $\mathrm{GaOx} / \mathrm{GaN}$ reflector through the PEC oxidation process. The PEC oxidized process occurred from the LS lines and formed the GaOx layers in the stack structure surrounding the mesa region.
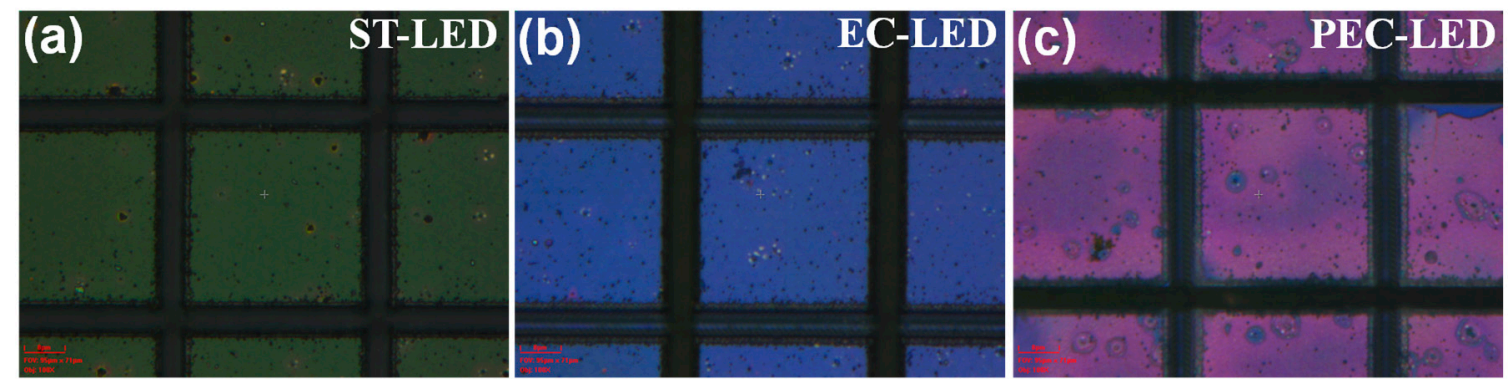

Figure 1. OM images of (a) ST-LED, (b) EC-LED, and (c) PEC-LED were observed with laser scribing line patterns. The mesa regions were $35 \times 35 \mu \mathrm{m}$.

In Figure 2a, eight pairs of $\mathrm{n}^{+}-\mathrm{GaN}: \mathrm{Si} / \mathrm{GaN}$ stack structures were transformed into a porous $\mathrm{GaN} / \mathrm{GaN}$ stack structure using the EC etching process. The pair thickness of $\mathrm{n}^{+}-\mathrm{GaN}: \mathrm{Si} / \mathrm{GaN}$ was about $102 \mathrm{~nm}$. After the PEC oxidation process, the porous GaN layer was oxidized as a GaOx layer, as shown in Figure 2b. The GaN layers in the PEC-LED structure became thinner compared to in the EC-LED structure, indicating that part of the GaN layer was oxidized during the PEC oxidation process. The porous $\mathrm{GaN}$ layers in the stack structure provided a channel for the $\mathrm{OH}^{-}$ions to oxidize 
the residual single crystalline $\mathrm{GaN}$ in the porous structure. The $\mathrm{GaOx} / \mathrm{GaN}$ stack structure was formed surrounding the mesa region through the lateral PEC oxidation process.

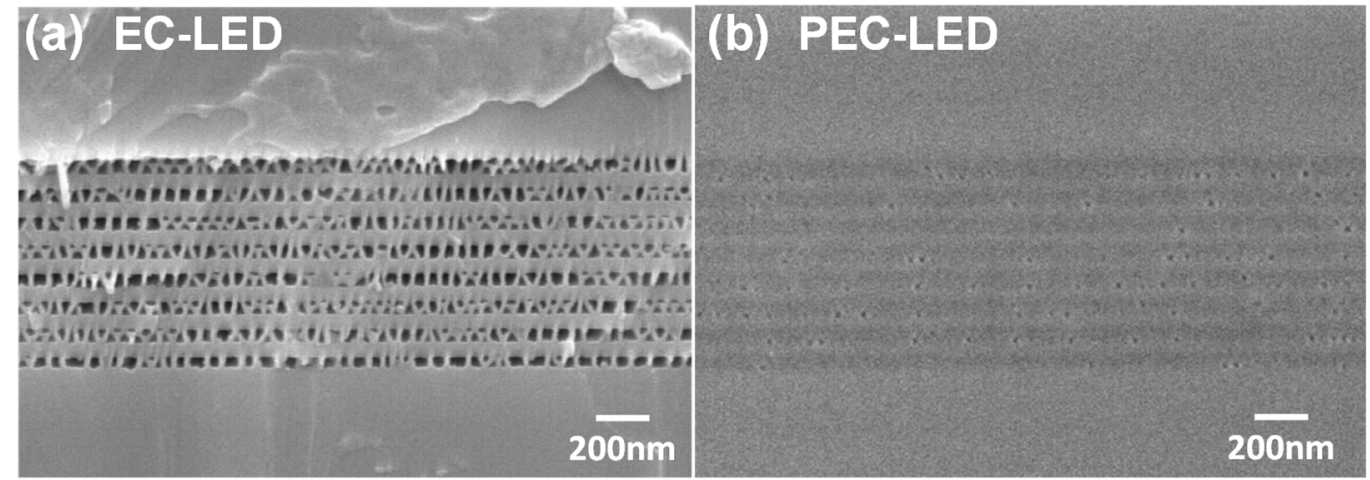

Figure 2. The SEM (scanning electron microscope) micrographs of (a) nanoporous GaN/GaN stack structure and (b) GaOx/GaN stack structure. The GaOx layer in the PEC-LED structure was prepared through the EC etching and PEC oxidized processes.

The EL (electroluminescence) emission images of the ST-LED, EC-LED, and PEC-LED are provided in Figure $3 a-c$, respectively, at an $18 \mu \mathrm{A}$ injection current. In Figure $3 a$, the uniform emission intensity was observed in the mesa region of the ST-LED. High-EL emission intensity was observed in the EC-LED structure caused by the formation of a high-reflectivity porous GaN reflector (Figure 3b). In the PEC-LED structure, the high-EL emission intensity was localized in the central mesa region as shown in Figure 3c. This occurred because the insulating GaOx layers in the stack structure formed surrounding the mesa region after the lateral PEC oxidation process. The EL light was emitted from the central mesa region and was extracted from the mesa sidewall region. The line EL intensity profiles of the LED samples are observed. After the PEC process, the EL emission intensity of the PEC-LED was localized in the central mesa region. An InGaN-based LED with GaOx current confinement structure was produced via the EC etching process and the PEC oxidation process.
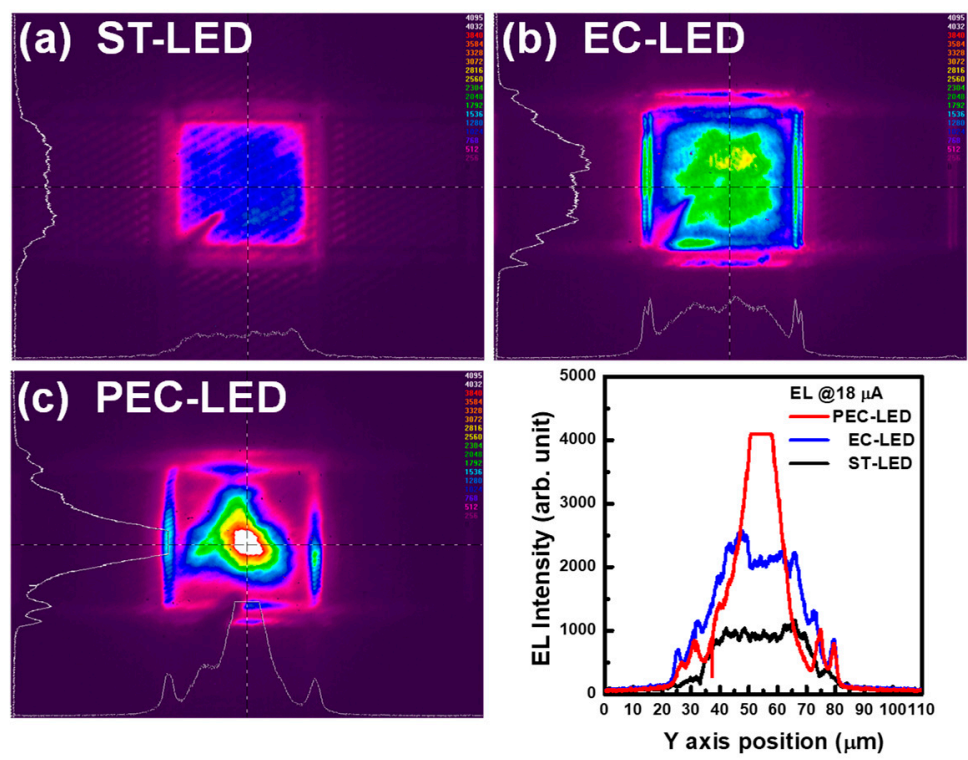

Figure 3. The EL emission images of (a) ST-LED, (b) EC-LED, and (c) PEC-LED observed using a beam profiler. The line intensity profile of the LED samples were measured at an $18 \mu \mathrm{A}$ operation current (see bottom right Figure). 
The reflectance spectra of the LED samples were measured as shown in Figure 4a. The light interference spectrum of the non-treated LED epi-structure was observed due to the light reflection between the top air/GaN:Mg and bottom GaN/sapphire interfaces. The reflectivity of the ST-LED was measured at about $20 \%$ as the reference spectrum. In the EC-LED structure, the high reflectance spectrum was observed at $469 \mathrm{~nm} / 80 \%$ and $446 \mathrm{~nm} / 76 \%$, which dipped at $456 \mathrm{~nm} / 62 \%$. After the EC etching process, a $0.7 \mu \mathrm{m}$-thick InGaN LED cavity was formed between the top air/GaN interface and the bottom porous $\mathrm{GaN}$ reflector. The dip wavelength in the reflectance spectrum was caused by the light interference in the short cavity structure. In the PEC-LED structure, the peak reflectance spectrum was observed at $464 \mathrm{~nm} / 69 \%$ and $439 \mathrm{~nm} / 66 \%$, which had a dip wavelength at $451 \mathrm{~nm} / 42 \%$. The central dip wavelength shifted from $456 \mathrm{~nm}$ (EC-LED) to $451 \mathrm{~nm}$ (PEC-LED) due to the transformation process from the porous $\mathrm{GaN}$ layers to the $\mathrm{GaOx}$ layers in the stack reflector structure. In Figure 4b, the EL peak wavelengths and linewidth were measured at $450 \mathrm{~nm}$ and $19.1 \mathrm{~nm}$ for the ST-LED, $448 \mathrm{~nm} / 17.2 \mathrm{~nm}$ for the EC-LED, and $453 \mathrm{~nm} / 16.5 \mathrm{~nm}$ for the PEC-LED structure, respectively, at a $3 \mathrm{~mA}$ operation current. The EL emission intensity of the EC-LED was higher than that of the ST-LED, caused by the formation of a highly reflective and conductive porous GaN reflector. The EL peak wavelength of the PEC-LED slightly redshifted compared to ST-LED, potentially caused by the thermal joule heat in the LED device at the high operating current density in the confinement aperture structure.
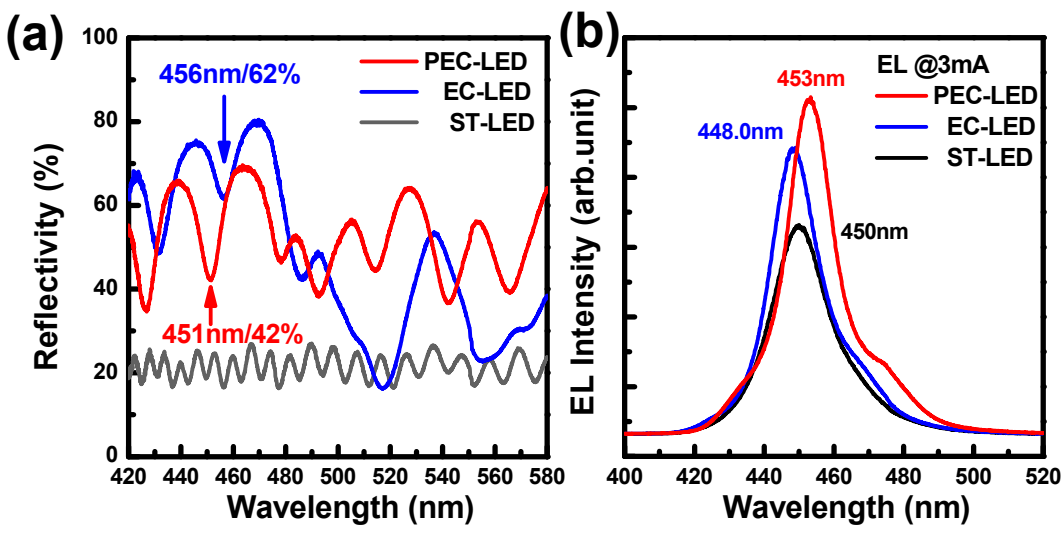

Figure 4. (a) The reflectance spectral of all LED structure and (b) the EL spectra of the LED samples measured with different embedded reflector spectra.

\section{Conclusions}

An InGaN LED structure with an embedded porous GaN reflector and a current-confined aperture was fabricated through the lateral EC etching and PEC oxidation processes. The central wavelength of the porous $\mathrm{GaN}$ reflector matched the EL emission spectrum. The porous GaN layers provided a large surface area for the following PEC oxidation process. The central wavelength of the reflectors was blueshifted by transforming the porous $\mathrm{GaN} / \mathrm{GaN}$ stack structure into a $\mathrm{GaOx} / \mathrm{GaN}$ stack structure. The injection current in the PEC-LED was confined to the aperture structure by being surrounded by insulating $\mathrm{GaOx}$ layers in the reflector structure. The produced PEC-LED structure with a porous GaN reflector and a current-confined aperture surrounding by insulating $\mathrm{GaOx}$ layers has the potential for nitride-based resonant-cavity LED device applications.

Author Contributions: Y.-Y.C., Y.-C.J. and C.-J.W. fabricated the EC-LED and PEC-LED. H.C. supporting the analysis of the LED samples. Y.-S.L. performed the EL measurement and analysis. C.-F.L. organized the experiment design and wrote the manuscript with contributed from other co-authors.

Funding: This research received no external funding. 
Acknowledgments: The authors gratefully acknowledge the financial support for this research by the Ministry of Science and Technology of Taiwan under grant No. 105-2221-E-005-012-MY2 and 107-2221-E-005-061. It was further supported by the Innovation and Development Center of Sustainable Agriculture's Featured Areas Research Center Program within the framework of Taiwan's Ministry of Education's Higher Education Sprout Project.

Conflicts of Interest: The authors declare no conflict of interest.

\section{References}

1. Nakamura, S. The Roles of Structural Imperfections in InGaN-Based Blue Light-Emitting Diodes and Laser Diodes. Science 1998, 281, 956-961. [CrossRef]

2. Someya, T.; Werner, R.; Forchel, A.; Catalano, M.; Cingolani, R.; Arakawa, Y. Room Temperature Lasing at Blue Wavelengths in Gallium Nitride Microcavities. Science 1999, 285, 1905-1906. [CrossRef] [PubMed]

3. Yang, G.M.; MacDougal, M.H.; Dapkus, P.D. Ultralow Threshold Current Vertical-cavity Surface-emitting Lasers Obtained with Selective Oxidation. Electron. Lett. 1995, 31, 886-888. [CrossRef]

4. Bissessur, H.K.; Koyama, F.; Iga, K. Modeling of Oxide-confined Vertical-cavity Surface-Emitting Lasers. IEEE J. Sel. Top. Quantum Electron. 1997, 3, 344-352. [CrossRef]

5. MacDougal, M.H.; Dapkus, P.D.; Bond, A.E.; Lin, C.-K.; Geske, J. Design and Fabrication VCSEL's with $\mathrm{Al}_{\mathrm{x}} \mathrm{O}_{\mathrm{y}}-\mathrm{GaAs}$ DBR's. IEEE J. Sel. Top. Quantum Electron. 1997, 3, 905-915. [CrossRef]

6. Dorsaz, J.; Bühlmann, H.J.; Carlin, J.F.; Grandjean, N.; Ilegems, M. Selective Oxidation of AlInN Layers for Current Confinement in III-nitride Devices. Appl. Phys. Lett. 2005, 87, 072102. [CrossRef]

7. Nakada, N.; Nakaji, M.; Ishikawa, H.; Egawa, T.; Umeno, M.; Jimbo, T. Improved characteristics of InGaN multiple-quantum-well light-emitting diode by GaN/AlGaN distributed Bragg reflector grown on sapphire. Appl. Phys. Lett. 2000, 76, 1804-1806. [CrossRef]

8. Lin, C.F.; Yao, H.H.; Lu, J.W.; Hsieh, Y.L.; Kuo, H.C.; Wang, S.C. Characteristics of Stable Emission GaN-Based Resonant-Cavity Light-Emitting Diodes. J. Cryst. Growth 2004, 261, 359-363. [CrossRef]

9. Yao, H.H.; Lin, C.F.; Kuo, H.C.; Wang, S.C. MOCVD Growth of AlN/GaN DBR Structures under Various Ambient Conditions. J. Cryst. Growth 2004, 262, 151-156. [CrossRef]

10. Carlin, J.F.; Ilegems, M. High-Quality AllnN for High Index Contrast Bragg Mirrors Lattice Matched to GaN. Appl. Phys. Lett. 2003, 83, 668-670. [CrossRef]

11. Lin, C.F.; Yang, Z.J.; Zheng, J.H.; Dai, J.J. Enhanced Light Output in Nitride-based Light-emitting Diodes by Roughening the Mesa Sidewall. IEEE Photonics Technol. Lett. 2005, 17, 2038-2040.

12. Lin, C.F.; Chen, K.T.; Huang, K.P. Blue Light-Emitting Diodes with an Embedded Native Gallium Oxide Pattern Structure. IEEE Electron Device Lett. 2010, 31, 1431-1433. [CrossRef] 